\title{
Die optimale Ausrichtung des Sonnenkollektors im Raum Zürich
}

\section{Einleitung}

In der jüngsten Vergangenheit hat das Interesse für Fragen der Sonnenenergie stark zugenommen. crundlegende Probleme bei Sonnenenergiekollektoren sind:

1. Welches ist die optimale Orientierung des Kollektors?

2. Wie gross ist die verfügbare Energie?

Der vorliegende Artikel möchte diese Fragen für Flachkollektoren beantworten.

\section{Theorie}

Das Studium der einfallenden kurzwelligen Strahlung auf geneigten Flächen wurde Ende des 19. Jahrhunderts aufgenommen. Die Berechnung der direkten Sonnenstrahlung ist eine geometrische Aufgabe, die bereits vor der Jahrundertwende gelöst werden konnte. Das einzige Problem war die Integration über die Zeit, die langwierige Berechnungen erfordert. Ohmura (1968) löste diese Aufgabe mit Hilfe von Vektoralgebra und numerischen Methoden. Die direkte Strahlung Q auf der Fläche mit Azimut A und Neigung B kann folgendermassen ausgedrückt werden: $\begin{aligned} Q & =I_{0}\left(\frac{r_{0}}{r}\right)^{2} q^{m}\left[\left(X_{1} \sin \rho \cos H-X_{2} \sin H+\right.\right. \\ & \left.\left.X_{3} \cos \varphi \cos H\right) \cos \delta+\left(-X_{1} \cos \rho+X_{3} \sin \rho\right) \sin \delta\right] 1 .\end{aligned}$

wobei $X_{1}=-\cos A \sin B ; X_{2}=\sin A \sin B$; $\mathrm{X}_{3}=\cos \mathrm{B} ; \mathrm{I}_{\mathrm{O}}=$ Sonnenkonstante, 1,95 $1 \mathrm{y} \mathrm{min}^{-1}$ oder 32,6 kWh $\mathrm{m}^{-2} \mathrm{~d}^{-1} ; \mathrm{r}=$ Abstand Erde - Sonne; $\mathrm{r}_{\mathrm{O}}=$ mittlerer Abstand Erde - Sonne; $\mathrm{q}=$ Durchsichtigkeit der Atmosphäre für die Sonnenstrahlung; $\rho=$ geographische Breite; $\delta=$ Deklination der Sonne; $\mathrm{H}=$ Stundenwinkel; $\mathrm{m}$ ist die optische Luftmasse, die, wenn die Sonnenhöhe grösser als $20^{\circ}$ ist, durch die folgende Formel angenähert werden kann:

$$
m=\frac{1}{\cos \rho \cos \delta \cos H+\sin \rho \sin \delta}
$$

Für niedrigen Sonnenstand ist Tabelle 137 in Smithsonian Meteorological Tables (List, 1966) zu empfehlen.

Die Tagessumme der direkten Strahlung ist das Integral der Gl. 1 über $\mathrm{H}$, von Sonnenaufgang bis
Sonnenuntergang auf der geneigten Fläche. Ein Intervall von 20 Minuten ist für die numerische Integration hinreichend. Die Genauigkeit des Resultats ist abhängig von jener der Schätzwerte der atmosphärischen Durchsichtigkeit q. Ohmuras Experiment (1969) zeigt, dass G1. 1 für eine mit 200 gegen Süden geneigte Fläche einen mittleren quadratischen Fehler von $181 \mathrm{y} \mathrm{d}-1$ ergibt, was $7 \%$ der tagestotalen direkten Strahlung entspricht.

Die mathematische Darstellung der diffusen Himmelsstrahlung für die geneigte Oberfläche wurde von Kondrat'yev (1965) entwickelt, unter der Annahme einer isotropischen und homogenen Himmelshaube. Er gelangte zur Formel:

$$
\mathrm{D}_{\mathrm{B}}=\mathrm{D}_{\mathrm{O}} \cos \frac{2 \mathrm{~B}}{2}
$$

$\mathrm{D}_{\mathrm{B}}$ : diffuse Hirmelsstrahlung auf der Neigung $\mathrm{B}$

$\mathrm{D}_{\mathrm{O}}$ : diffuse Himmelsstrahlung auf der horizontalen Fläche.

Die Forme1 von Kondrat'yev ergibt eine systematische Unterbewertung bei Südhängen und eine Ueberbewertung bei Nordhängen. Bei $20^{\circ}$ Neigung beträgt diese Ueber- bzw. Unterbewertung etwa $8 \%$ der Tageswerte.

Die geneigte Fläche empfängt normalerweise reflektierte diffuse Strahlung $\mathrm{R}_{\mathrm{B}}$ aus der Ungebung. Diese Komponente wurde von Garnier und Ohmura (1970) folgendermassen dargestellt:

$$
R_{B}=r G \sin \frac{2}{2}
$$

r: mittlere Reflektivität der Umgebung

G: Globalstrahlung auf der horizontalen Fläche.

Der Beitrag dieser Strahlungskomponente ist bedeutend kleiner als jener der anderen beiden.

Die totale einfallende kurzwellige Strahlung (Globalstrahlung) auf der geneigten Fläche ist die Summe der Gleichung 1,2 und 3. Der mittlere quadratische Fehler bei einer mit $20^{\circ}$ gegen Süden geneigten Fläche beträgt $8 \%$ der mittleren täglichen Globalstrahlung.

Dr. Atsumu Ohmura, Geographisches Institut ETH, Sonneggstrasse 5, 8006 Zürich 
Tab.1:

Monatsmittelstrahlung für Zürich-Kloten nach Schram und Thams (1970), ly d $\mathrm{d}^{-1}$ Messjahre 1964-1967

\begin{tabular}{|l|rrrrrrrrrrrr|c|}
\hline & Jan & Feb & Mär & Apr & Mai & Jun & Jul & Aug & Sep & Okt & Nov & Dez & $\begin{array}{c}\text { Jalırestotal } \\
\text { kly } y^{-1}\end{array}$ \\
\hline $\begin{array}{l}\text { direkte Strahlung } \\
\text { diffuse Himmels- }\end{array}$ & 58 & 64 & 89 & 187 & 241 & 278 & 285 & 202 & 153 & 72 & 18 & 3 & 134 \\
$\begin{array}{l}\text { strahlung } \\
\text { globale Strahlung }\end{array}$ & 70 & 146 & 221 & 358 & 447 & 498 & 501 & 393 & 292 & 167 & 74 & 54 & 268 \\
$\begin{array}{l}\text { reflektierte Strah- } \\
\text { lung (10\% Albedo) } \\
\begin{array}{l}\text { reflektierte Strah- } \\
\text { lung (50\% Albedo) }\end{array}\end{array}$ & 75 & 15 & 22 & 36 & 45 & 50 & 50 & 39 & 29 & 17 & 7 & 5 & 27 \\
\hline
\end{tabular}

Tab.2:

Direkte, himmelsdiffuse, reflektierte diffuse und globale Strahlung auf die Südhänge für

vier Jahreszeiten und ein Jahr, Einheit für Monatswerte in $1 y^{\prime} d^{-1}$ und Jahreswerte lily $y^{-1}$

\begin{tabular}{|c|c|c|c|c|c|c|c|c|c|c|}
\hline Neigung in Grad & $0^{\circ}$ & $10^{\circ}$ & $20^{\circ}$ & $30^{\circ}$ & $40^{\circ}$ & $50^{\circ}$ & $60^{\circ}$ & $70^{\circ}$ & $80^{\circ}$ & $90^{\circ}$ \\
\hline \multicolumn{11}{|l|}{ März } \\
\hline $\begin{array}{l}\text { direkte Strahlung } \\
\text { himmelsdiffuse Strahlung } \\
\text { reflektierte diffuse Strahlung } \\
\text { globale Strahlung }\end{array}$ & $\begin{array}{r}89 \\
132 \\
0 \\
221\end{array}$ & $\begin{array}{r}106 \\
131 \\
0 \\
237\end{array}$ & $\begin{array}{r}120 \\
128 \\
1 \\
249\end{array}$ & $\begin{array}{r}130 \\
123 \\
2 \\
255\end{array}$ & $\begin{array}{r}136 \\
116 \\
3 \\
255\end{array}$ & $\begin{array}{r}138 \\
108 \\
4 \\
250\end{array}$ & $\begin{array}{r}136 \\
99 \\
6 \\
241\end{array}$ & $\begin{array}{r}130 \\
88 \\
7 \\
225\end{array}$ & $\begin{array}{r}120 \\
78 \\
9 \\
207\end{array}$ & $\begin{array}{r}106 \\
66 \\
11 \\
183\end{array}$ \\
\hline \multicolumn{11}{|l|}{ Juni } \\
\hline $\begin{array}{l}\text { direkte Strahlung } \\
\text { himmelsdiffuse Strahlung } \\
\text { reflektierte diffuse Strahlung } \\
\text { globale Strahlung }\end{array}$ & $\begin{array}{r}278 \\
220 \\
0 \\
498\end{array}$ & $\begin{array}{r}287 \\
218 \\
0 \\
505\end{array}$ & $\begin{array}{r}288 \\
213 \\
2 \\
503\end{array}$ & $\begin{array}{r}280 \\
205 \\
3 \\
488\end{array}$ & $\begin{array}{r}265 \\
194 \\
6 \\
465\end{array}$ & $\begin{array}{r}242 \\
181 \\
9 \\
432\end{array}$ & $\begin{array}{r}212 \\
165 \\
13 \\
390\end{array}$ & $\begin{array}{r}177 \\
148 \\
16 \\
341\end{array}$ & $\begin{array}{r}137 \\
129 \\
21 \\
287\end{array}$ & $\begin{array}{r}95 \\
110 \\
25 \\
230\end{array}$ \\
\hline \multicolumn{11}{|l|}{ September } \\
\hline $\begin{array}{l}\text { direkte Strahlung } \\
\text { himmelsdiffuse Strahlung } \\
\text { reflektierte diffuse Strahlung } \\
\text { globale Strahlung }\end{array}$ & $\begin{array}{r}153 \\
139 \\
0 \\
292\end{array}$ & $\begin{array}{r}182 \\
138 \\
0 \\
320\end{array}$ & $\begin{array}{r}206 \\
135 \\
1 \\
345\end{array}$ & $\begin{array}{r}224 \\
129 \\
2 \\
355\end{array}$ & $\begin{array}{r}234 \\
122 \\
4 \\
360\end{array}$ & $\begin{array}{r}238 \\
114 \\
5 \\
357\end{array}$ & $\begin{array}{r}234 \\
104 \\
7 \\
345\end{array}$ & $\begin{array}{r}224 \\
93 \\
10 \\
327\end{array}$ & $\begin{array}{r}206 \\
82 \\
12 \\
300\end{array}$ & $\begin{array}{r}182 \\
70 \\
15 \\
267\end{array}$ \\
\hline \multicolumn{11}{|l|}{$\underline{\text { Dezember }}$} \\
\hline direkte Strahlung & 8 & 13 & 18 & 22 & 26 & 28 & 30 & 31 & 31 & 30 \\
\hline himmelsdiffuse Strahlung & 46 & 46 & 45 & 43 & 41 & 38 & 35 & 31 & 27 & 23 \\
\hline $\begin{array}{l}\text { reflektierte diffuse Strahlung } \\
\text { (ohne Schneedecke) }\end{array}$ & 0 & 0 & 0 & 0 & 1 & 1 & 2 & 2 & 2 & 3 \\
\hline $\begin{array}{l}\text { reflektierte diffuse Strahlung } \\
\text { (Schneedecke) }\end{array}$ & 0 & 0 & 1 & 2 & 3 & 5 & 7 & 9 & 11 & 14 \\
\hline globale Strahlung ohne Schnee & 54 & 59 & 63 & 65 & 68 & 67 & 67 & 64 & 60 & 56 \\
\hline globale Strahlung mit Schnee & 54 & 59 & 64 & 67 & 70 & 71 & 72 & 71 & 69 & 67 \\
\hline \multicolumn{11}{|l|}{ Jahressumme } \\
\hline $\begin{array}{l}\text { globale Strahlung ohne } \\
\text { Winterschnee }\end{array}$ & 97 & 102 & 106 & 106 & 105 & 101 & 95 & 87 & 78 & 67 \\
\hline $\begin{array}{l}\text { globale Strahlung mit } \\
\text { Winterschnee }\end{array}$ & 97 & 102 & 106 & 106 & 105 & 101 & 96 & 88 & 79 & 68 \\
\hline
\end{tabular}




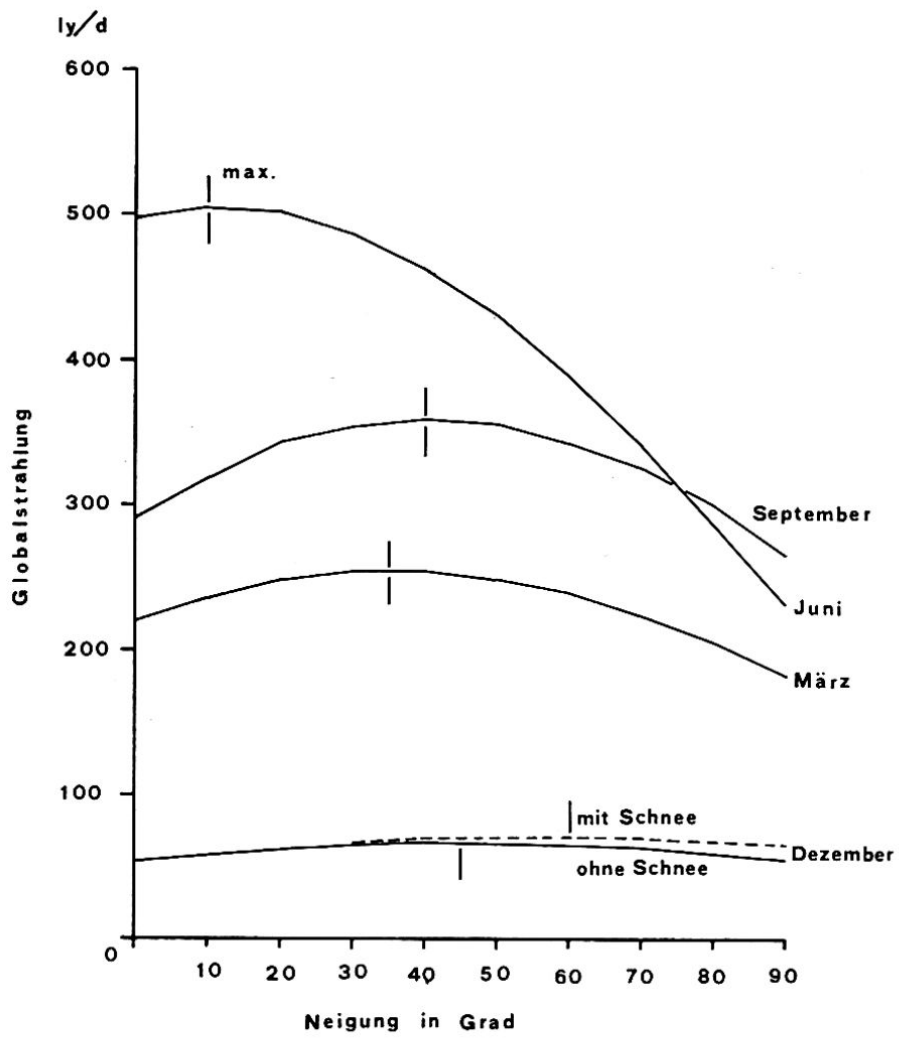

Fig.1: Monatsmittelglobalstrahlung auf den Südhängen im Gebiet zürich

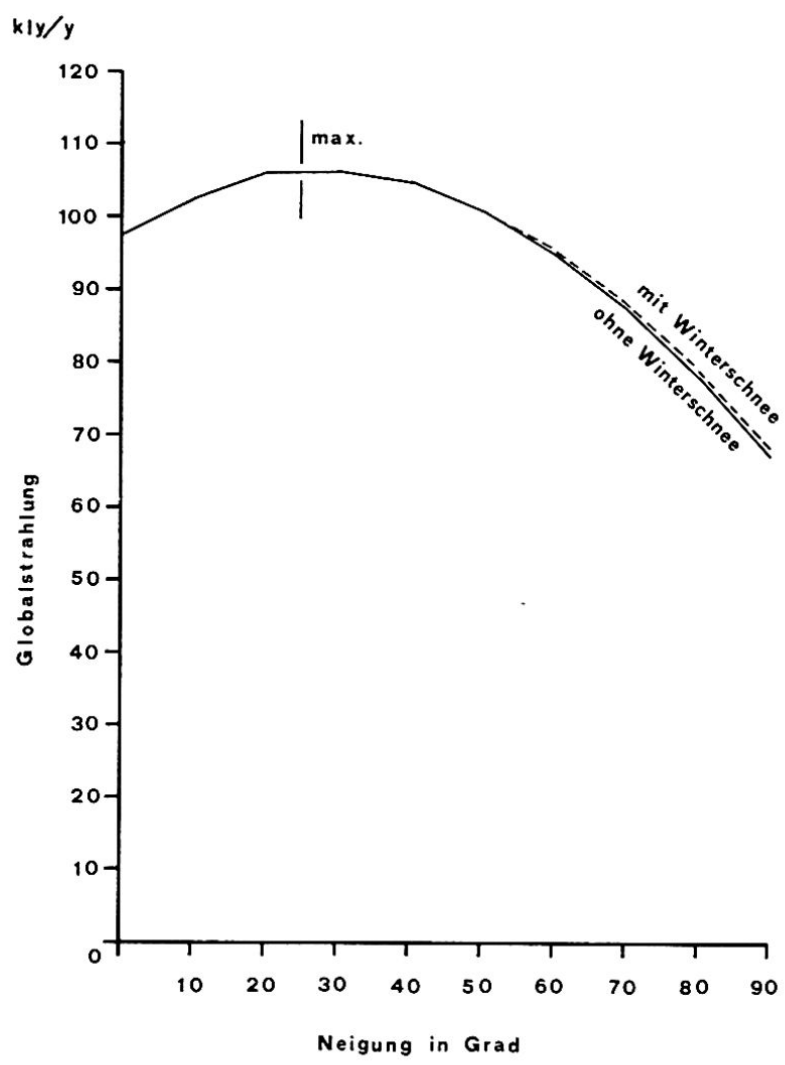

Fig.2: Jahrestotalglobalstrahlung auf den Südhängen im Gebiet zürich

\section{Klimatologische Daten}

Die besten und zugleich die am leichtesten zugänglichen Daten sind jene von Schram und Thams (1970). Diese beiden Autoren fassten Strahlungsdaten für Zürich-Kloten aus 10 Jahren zusammen und verglichen sie mit den Messungen an der MZA in Zürich. Es zeigte sich, dass die Monatsmittel der Globalstrahlung der beiden Stationen identisch sind. Es ist deshalb gerechtfertigt, die Daten von Kloten auf Zürich anzuwenden. Die Klotener Daten haben den Vorteil, dass dort die Globalstrahlung und die, diffuse Himmelsstrahlung während vier Jahren gleichzeitig erfasst wurden, was für unsere Zwecke lang genug ist. Die Monatswerte der direkten, himmelsdiffusen und globalen Strahlung werden in Tabelle 1 gegeben.

\section{Berechnung}

In den gemässigten Breiten der Nordhemisphäre empfangen die Südhänge $\left(A=180^{\circ}\right)$ immer die grösste Globalstrahlung. Deshalb wurde Gl. 1 nur auf $\mathrm{A}=180^{\circ}$ angewendet. Für je $10^{\circ}$ Neigung wird die Globalstrahlung für März, Juni, September und Dezember berechnet. Die Albedo der Umgebung wird mit $10 \%$ angenommen, was für überbaute Gebiete ein vernünftiger Wert ist. Die Albedo der Schneedecke wird mit $50 \%$ angenähert.

\section{Ergebnisse}

Die Resultate der Berechnung werden in Tabelle 2 und in den Abbildungen 1 und 2 dargestellt. Die optimale Neigung variiert mit der Jahreszeit, von $10^{\circ}$ im Juni bis $40^{\circ}$ zu $60^{\circ}$ im Dezember. Die Neigungsabhängigkeit der Strahlung nimmt mit der Deklination stark ab. Die optimale feste Neigung von $25^{\circ}$ ergibt eine jährliche Globalstrahlung von 106,2 kly $\mathrm{y}^{-1}\left(1231 \mathrm{kWh} \mathrm{m}^{-2}\right.$ $\left.\mathrm{y}^{-1}\right)^{*}, 9 \mathrm{kly}\left(104 \mathrm{kWh} \mathrm{m}^{-2}\right)$ mehr als der horizontale Kollektor. Lässt man den Neigungswinkel mit der Jahreszeit optimal variieren, so gewinnt man dadurch bloss 2,3 kly (27 $\left.\mathrm{kWh} \mathrm{m}^{-2}\right)$.

Oft ist die Neigung des Kollektors durch die Dachschräge gegeben. Ein nach Süden gerichtetes Dach mit der hierzulande üblichen Schräge von etwa $35^{\circ}$ empfängt jährlich 105,5 kly(1223 kWh $\mathrm{m}^{-2}$ ), also fast den Maximalwert. Weicht das Azimut des Daches $45^{\circ}$ von Süden ab, so muss mit einer Strahlungsreduktion von $6 \mathrm{kly} \mathrm{y}^{-1}(70 \mathrm{kWh}$ $\mathrm{m}^{-2} \mathrm{y}^{-1}$ ) gerechnet werden.

* In der Schweiz beträgt der Energiebedarf pro Kopf ca. $9000 \mathrm{kWh}$ pro Jahr. Unter der Annahme eines Falchkollektors mit 50 \% Wirkungsgrad braucht man für diesen Bedarf $16 \mathrm{~m}^{2}$ Kollektorfläche pro Person, oder eine solche von $100 \mathrm{~km}^{2}$ für die Gesamtbevölkerung. Dies ist keine unrealistische $\mathrm{Zahl}$. 
Mein Dank richtet sich an Prof. Dr. F. Müller vom Geographischen Institut ETH, der mein Strahlungsstudium seit vielen Jahren unterstützt. Dankbar bin ich auch Herrn K. Scherler, der dieses Manuskript gelesen und lesbar gemacht hat.

\section{Literatur}

GARNIER, B.J. and OHMURA, A.: The Evaluation of Surface Variations of Short-wave Radiation Income, Solar Energy, Vol.13, 1970, pp.2134.

KONDRAT'YEV, K.Ya.: Radiative Heat Exchange in the Atmosphere (übersetzt aus dem Russischen von 0. Tedder), Pergamon Press, Oxford and New York, 1965, p.332.

LIST, R.J.: Smithsonian Meteorological Tables, Smithsonian Misc. Col1., Vo1.114, 6th Edn, 1966, pp.422.

OHMURA, A.: The Computation of Direct Insolation on a Slope, Climatological Bulletin, No.3, McCill University, Montreal, 1968, pp.42-53.

OHMURA, A.: Computation and Mapping the Shortwave Radiation on a Slope, M.Sc. Thesis, McGill University, Montrea1, 1969

SCHRAM, K. und THAMS, J.C.: Die kurzwellige Globalstrahlung und die diffuse Himmelsstrahlung auf dem Flugplatz Zürich-Kloten, Veröffentlichungen der Schweiz. Meteorolog. Zentralanstalt, Nr.16, 1970, S.18.

\section{Literaturbesprechung}

BOEGLI, Alfred: Karsthydrographie und physische Speläologie. 292 S., $160 \mathrm{Abb}$. und 12 Tafeln, Springer-Verlag, Berlin, 1978, ISBN 3-540-09015. DM 58,-

Höhlen und die Hydrographie der Karstgebiete sind zur Zeit ein sehr aktuelles Forschungsgebiet. Die Forschung wird aus unterschiedlichen Motiven betrieben. Auf der einen Seite sind es mehr sportlich orientierte Höhlenforscher, die in schwierigsten Befahrungen Sachverhalte über kaum zugängliche Höhlensysteme und ihre Hydrographie erkunden. Andererseits sind es an Problemen der wissenschaftlichen Grundlagen bis hin $\mathrm{zu}$ aktuellen Umweltfragen arbeitende Wissenschaftler, die den unterirdischen Karst untersuchen und denen es oft an Erfahmmg vor Ort fehlt. Nur sehr wenige vereinen die Eigenschaften des aktiven Höhlenforschers mit der des Wissenschaftlers, wie dies bei A. BOEGLI der Fall ist. Seit nunmehr über 30 Jahren in Höhlen forschend, hat er eine Vielzahl von Einzelpublikationen über das Karstwasser, Lösungsvorgänge in Karst und Höhlen vorgelegt. Weiterhin wurden auf Fachtagungen neue Thesen zur Karsthydrographie und Speläologie vorgetragen. Diese zusammen fanden Eingang in das vorliegende Buch, das nicht nur bisherige Forschungsergebnisse in klarer sachlicher Art zusammenfasst und nur vorgetragene neue Theorien in naturwissenschaftlich abgesicherter Breite erstmals in fixierter Form vorlegt, sondern auch die gesamten Phänomene des unterirdischen Karstes behandelt. In einzelnen Kapiteln werden dargestellt:

Verkarstungsfähige Gesteine. - Lösungsvorgänge an verkarstungsfähigen Gesteinen, Korrosion. Uebersicht über den Exokarst. - Endokarst und Karsthydrographie. - Physikalisches Verhalten der Karstwässer. - Die karsthydrographischen Zonen. - Karstwasser - Grundwasser. - Unterirdische Karstniveaus. - Karstquellen. - Markierungsstoffe. - Inkasion, - Speläomorphologie, die Formenwelt der unterirdischen Abtragung. Höhlensedimente. - Speläogenese. - Speläometeorologie. - Speläoklimatologie. - Eishöhlen. Klassifikation der unterirdischen Hohlräume.Höhlensignaturen.

Die Einführungskapitel über Petrographie und Exokarst sind sehr knapp gehalten, sie geben aber einen Ueberblick über den aktuellen Stand der Karstforschung. Die Lösungsvorgänge sind ausführlich dargeste11t und Einzelprozesse methodisch sehr geschickt durch verständliche $\mathrm{Ab}-$ bildungen erläutert. Erfreulicherweise finden hierbei Forschungsergebnisse sowohl aus dem anglo-amerikanischen als auch aus dem französischen Sprachbereich Eingang. Vielleicht wäre es für den mit der physikalischen und chemischen Fachliteratur nicht so vertrauten Leser gut gewesen, einige Standardwerke anzuführen, um die Grundlagen für die dargelegten Kapitel leichter zu erarbeiten.

Die Stärke des Buches liegt aber in den Kapiteln über den unterirdischen Karst, seiner Höhlen und seiner Wässer. Hier zeigen sich einerseits die Erfahrungen von BOEGLI, andererseits tragen die guten Kontakte von BOEGLI zu anderen Höhlenforschern gute Früchte, da sonst kaum bekannte Sachverhalte eine weite Verbreitung finden und zur Unterstützung wissenschaftlicher Theorien dienen.

Die klare Darstellung und naturwissenschaftliche Untermauerung der Sachverhalte sowie deren Ableitung, verbunden mit methodisch sehr guten Dokumentationen, weist den Autor als erfahrenen Hochschullehrer aus. Das Buch ist ein Meilenstein der Karstforschung, es wird auf Jahre Grundlage für hydrogranhische und speläologische Untersuchungen sein und zu neuen Arbeiten anregen.

Es kann allen an der Karst- und Höhlenforschung interessierten - besonders den Geowissenschaftlern - nur empfohlen werden.

GH $3 / 79$ Karl-Heinz Pfeffer, Köln 